\title{
On Vervaat and Vervaat-error type processes for partial sums and renewals
}

\author{
Endre Csáki ${ }^{1}$ \\ Alfréd Rényi Institute of Mathematics, Hungarian Academy of Sciences, Budapest, P.O.B. 127, \\ H-1364, Hungary. E-mail: csaki@renyi.hu \\ Miklós Csörgö2 \\ School of Mathematics and Statistics, Carleton University, Ottawa, Ontario, Canada K1S $5 B 6$. \\ E-Mail: mcsorgo@math.carleton.ca \\ Zdzisław Rychlik ${ }^{3}$ \\ Instytut Matematyki, Uniwersytet Marii Curie-Skłodowskiej, pl. Marii Curie-Skłodowskiej 1, \\ PL-20 031 Lublin, Poland. E-Mail: rychlik@golem.umcs.lublin.pl \\ Josef Steinebach ${ }^{4}$ \\ Universität zu Köln, Mathematisches Institut, Weyertal 86-90, D-50 931 Köln, Germany. \\ E-Mail: jost@math.uni-koeln.de
}

\begin{abstract}
We study the asymptotic behaviour of stochastic processes that are generated by sums of partial sums of i.i.d. random variables and their renewals. We conclude that these processes cannot converge weakly to any nondegenerate random element of the space $D[0,1]$. On the other hand we show that their properly normalized integrals as Vervaat-type stochastic processes converge weakly to a squared Wiener process. Moreover, we also deal with the asymptotic behaviour of the deviations of these processes, the so-called Vervaat-error type processes.
\end{abstract}

Keywords. Partial sums, renewals, Vervaat and Vervaat-error type processes, Wiener process, strong and weak approximations, weak convergence.

2000 Mathematics Subject Classification. Primary 60F17; secondary 60F05;60F15

\footnotetext{
${ }^{1}$ Research supported by the Hungarian National Foundation for Scientific Research, Grant No. T 037886 and T 043037.

${ }^{2}$ Research supported by an NSERC Canada Grant at Carleton University, Ottawa.

${ }^{3}$ Research supported by the Deutsche Forschungsgemeinschaft through the German-Polish project 436 POL 113/98/0-1 "Probability measures".

${ }^{4}$ Research supported by a Polish-German Exchange Grant No. BWM-III/DAAD/801/JK99, and by a special grant from Marie Curie-Skłodowska University, Lublin.
} 


\section{Introduction}

Let $X_{1}, X_{2}, \ldots$ be an i.i.d. sequence with d.f. $F(x)=P\left(X_{1} \leq x\right),-\infty<x<\infty$, and

(i) $E X_{1}=\mu>0$;

(ii) $0<\operatorname{Var}\left(X_{1}\right)=\sigma^{2}(<\infty)$;

(iii) $E X_{1}^{4}<\infty$

Set $S_{n}:=X_{1}+\cdots+X_{n}(n=1,2, \ldots), S_{0}:=0$. For $t \geq 0$ define pointwise the corresponding renewal (or first passage time) process as $N(t):=\min \left\{n \geq 1: S_{n}>t\right\}$. We further introduce the "average" and "standardized" processes

$$
\begin{gathered}
S_{n}(t):=S_{[n t]} /(n \mu) \quad(t \geq 0) ; \\
s_{n}(t):=n^{1 / 2} \sigma^{-1} \mu\left(S_{n}(t)-t\right)=n^{-1 / 2} \sigma^{-1}\left(S_{[n t]}-n \mu t\right) \quad(t \geq 0) ; \\
N_{n}(t):=N(n \mu t) / n \quad(t \geq 0) ; \\
r_{n}(t):=n^{1 / 2} \sigma^{-1} \mu\left(N_{n}(t)-t\right)=n^{-1 / 2} \sigma^{-1}(\mu N(n \mu t)-n \mu t) \quad(t \geq 0),
\end{gathered}
$$

where $[x]$ denotes the integer part of $x$.

Throughout the paper $\|\cdot\|$ denotes the sup-norm on $D[0,1]$, i.e., for $f \in D[0,1],\|f\|:=$ $\sup _{0 \leq t \leq 1}|f(t)|$.

We first collect some well-known facts concerning the above processes.

Theorem A On a large enough probability space, for $X_{1}, X_{2}, \ldots$ satisfying (i), (ii) and (iii) above, one can construct a standard Wiener process $\{W(t): 0 \leq t<\infty\}$, such that, the processes $s_{n}$ and $r_{n}$ can be simultaneously approximated by $W$ as follows:

$$
\lim _{n \rightarrow \infty} n^{1 / 4}\left\|s_{n}-W_{n}\right\|=0 \quad \text { a.s. }
$$

and

$$
\limsup _{n \rightarrow \infty} n^{1 / 4}(\log n)^{-1 / 2}(\log \log n)^{-1 / 4}\left\|r_{n}+W_{n}\right\|=2^{1 / 4} \sigma^{1 / 2} \mu^{-1 / 2} \quad \text { a.s. }
$$

where $W_{n}(t):=n^{-1 / 2} W(n t)$.

The result (1.5) is due to Komlós et al. (1976), and that of (1.6) is due to Horváth (1984). For more details and further developments we refer to Chapter 2 of Csörgö and Horváth (1993).

Consider now the stochastic process

$$
R_{n}^{*}(t):=s_{n}(t)+r_{n}(t), \quad 0 \leq t \leq 1,
$$

which can be viewed as the remainder term in the Bahadur-Kiefer type representation $r_{n}=-s_{n}+R_{n}^{*}$ of the renewal process $r_{n}$ in terms of the partial sums process $s_{n}$. In particular, Bahadur (1966), Kiefer $(1967,1970)$ introduced and studied the stochastic process

$$
R_{n}(t):=\alpha_{n}(t)+\beta_{n}(t), \quad 0 \leq t \leq 1
$$


that is known in the literature as the Bahadur-Kiefer process, where

$$
\begin{aligned}
& \alpha_{n}(t):=n^{1 / 2}\left(F_{n}(t)-t\right), \quad 0 \leq t \leq 1, \\
& \beta_{n}(t):=n^{1 / 2}\left(F_{n}^{-1}(t)-t\right), \quad 0 \leq t \leq 1,
\end{aligned}
$$

the uniform empirical and quantile processes, with $F_{n}^{-1}$ being the left-continuous inverse of the rightcontinuously defined empirical distribution function $F_{n}$ of the independent uniform $(0,1)$ random variables $U_{1}, \ldots, U_{n}, n \geq 1$.

In this regard, we summarize the most relevant results of Kiefer $(1967,1970)$ in the following theorem.

Theorem B For every fixed $t \in(0,1)$, we have

$$
\begin{aligned}
n^{1 / 4} R_{n}(t) & \stackrel{\mathcal{D}}{\rightarrow}(t(1-t))^{1 / 4} \mathcal{N}(|\tilde{\mathcal{N}}|)^{1 / 2}, \quad n \rightarrow \infty, \\
\limsup _{n \rightarrow \infty} \frac{n^{1 / 4}\left|R_{n}(t)\right|}{(\log \log n)^{3 / 4}} & =(t(1-t))^{1 / 4} \frac{2^{5 / 4}}{3^{3 / 4}} \quad \text { a.s. },
\end{aligned}
$$

where $\mathcal{N}$ and $\widetilde{\mathcal{N}}$ are independent standard normal variables and $\stackrel{\mathcal{D}}{\rightarrow}$ denotes convergence in distribution. Also,

$$
\lim _{n \rightarrow \infty} n^{1 / 4}(\log n)^{-1 / 2} \frac{\left\|R_{n}\right\|}{\left(\left\|\alpha_{n}\right\|\right)^{1 / 2}}=1 \quad \text { a.s. }
$$

As to (1.10), Kiefer (1970) announced it but proved only convergence in probability (cf. Theorem 1A, and the two sentences right after, in Kiefer, 1970). The upper bound for the almost sure convergence in (1.10) was proved by Shorack (1982), and the lower bound by Deheuvels and Mason (1990).

Concerning similar known results for the process $R_{n}^{*}$, we summarize them in the next theorem.

Theorem C Under the assumptions (i), (ii), (iii) we have for every fixed $t \in(0,1]$

$$
n^{1 / 4} R_{n}^{*}(t) \stackrel{\mathcal{D}}{\rightarrow} t^{1 / 4} \sigma^{1 / 2} \mu^{-1 / 2} \mathcal{N}(|\widetilde{\mathcal{N}}|)^{1 / 2}, \quad n \rightarrow \infty .
$$

where, as in Theorem $\mathrm{B}, \mathcal{N}$ and $\widetilde{\mathcal{N}}$ are independent standard normal random variables, and

$$
\lim _{n \rightarrow \infty} n^{1 / 4}(\log n)^{-1 / 2}\left\|R_{n}^{*}\right\| /\left\|r_{n}\right\|^{1 / 2}=\sigma^{1 / 2} \mu^{-1 / 2} \quad \text { a.s. }
$$

For the result in (1.11) we refer to Csörgő and Horváth (1993, Theorem 2.1.5) and for that of (1.12) to Deheuvels and Mason (1990, Theorem 1B).

As a consequence of (1.12), on account of

$$
\left.\left\|r_{n}\right\| \stackrel{\mathcal{D}}{\rightarrow}\|W\| \quad \text { (cf., e.g., Vervaat }(1972)\right),
$$

as $n \rightarrow \infty$, we have (cf. Deheuvels and Mason (1990))

$$
n^{1 / 4}(\log n)^{-1 / 2}\left\|R_{n}^{*}\right\| \stackrel{\mathcal{D}}{\rightarrow} \sigma^{1 / 2} \mu^{-1 / 2}\|W\|
$$


Based on (1.11) and (1.14), the next conclusion is immediate.

Corollary 1.1 Given the assumptions (i), (ii), (iii), the statement

$$
a_{n} R_{n}^{*} \stackrel{\mathcal{D}}{\rightarrow} Y, \quad n \rightarrow \infty
$$

cannot hold true in the space $D[0,1]$ (endowed with the Skorokhod topology) for any non-degenerate random element $Y$ of $D[0,1]$ with any normalizing sequence $\left\{a_{n}\right\}$.

The respective results of (1.11) and (1.12) are based on a strong invariance principle for the Bahadur-Kiefer type process $\left\{R_{n}^{*}(t), 0 \leq t \leq 1 ; n=1,2, \ldots\right\}$ that was explicitly stated in Csörgö and Horváth (1993, p. 43) as follows.

Theorem D On assuming the conditions (i), (ii), (iii), on the probability space of Theorem A, as $n \rightarrow \infty$, we have

$$
R_{n}^{*}(t)=n^{-1 / 2}\left(W(n t)-W\left(n t-\frac{\sigma}{\mu} W(n t)\right)\right)+o\left(n^{-1 / 4}\right) \quad \text { a.s. },
$$

uniformly in $t \in[0,1]$.

Corollary 1.1 and Theorem 3 of Vervaat (1972) serve as motivation for studying the following Vervaat-type process for partial sums and renewals:

$$
\begin{aligned}
V_{n}(t) & :=\int_{0}^{t}\left\{\left(S_{n}(s)-s\right)+\left(N_{n}(s)-s\right)\right\} d s \\
& =\int_{0}^{t} M_{n}(s) d s, \quad 0 \leq t \leq 1,
\end{aligned}
$$

where $M_{n}(s):=n^{-1 / 2} \sigma \mu^{-1} R_{n}^{*}(s), s \geq 0$.

So far we have been dealing with renewal processes of partial sums under the conditions (i), (ii) and (iii), that is to say we had general renewal processes in mind. We will now see that the just introduced Vervaat-type process $V_{n}$ will be well-behaving asymptotically as $n \rightarrow \infty$. However the asymptotic behaviour of $V_{n}$ will be different when it is based on general renewal processes, as compared to it being based on ordinary renewal processes, i.e., when in addition to (i), (ii), (iii), we also assume that $X_{1}$ is positive. Consequently, in our Section 2 we will deal with Vervaat-type processes for ordinary renewals, while Section 3 will be devoted to studying such processes for general renewals. Moreover, in Section 4 we will also be studying the asymptotic behaviour of Vervaat errortype processes that, based on the respective results of Sections 2 and 3, will be defined there (cf. (4.1)).

\section{The Vervaat-type process for ordinary renewals}

In addition to the conditions (i), (ii), (iii) of Section 1, in this Section we assume also the condition (iv) $P\left(X_{1}>0\right)=1$. 
Theorem 2.1 Assume the conditions (i), (ii), (iii) and (iv). Then, as $n \rightarrow \infty$

$$
\frac{n}{\log \log n}\left\|V_{n}-\frac{1}{2} \bar{S}_{n}^{2}\right\| \rightarrow 0 \quad \text { a.s. }
$$

and

$$
n\left\|V_{n}-\frac{1}{2} \bar{S}_{n}^{2}\right\| \stackrel{P}{\rightarrow} 0
$$

where $\bar{S}_{n}(t):=S_{n}(t)-t$.

Proof. Given condition (iv) it can be checked (cf. Figure 1 below) that $V_{n}$ has the following representation:

$$
V_{n}(t)=A_{n}(t)-\bar{S}_{n}(t) \bar{N}_{n}(t)-\frac{1}{2} \bar{N}_{n}^{2}(t),
$$

where

$$
\begin{gathered}
A_{n}(t):=\int_{N_{n}(t)}^{t}\left(\bar{S}_{n}(s)-\bar{S}_{n}(t)\right) d s, \\
\bar{S}_{n}(t):=S_{n}(t)-t=(n \mu)^{-1}\left(S_{[n t]}-n \mu t\right), \\
\bar{N}_{n}(t):=N_{n}(t)-t=n^{-1}(N(n \mu t)-n t),
\end{gathered}
$$

for all $0 \leq t \leq 1$.

In order to check the above representation, we note that

$$
N(n \mu t)=k, \quad \text { for } \quad \frac{S_{k-1}}{n \mu} \leq t<\frac{S_{k}}{n \mu} .
$$

Also, by calculations, we arrive at

$$
\begin{aligned}
\int_{0}^{t} & \left(S_{n}(s)+N_{n}(s)\right) d s=t^{2}+\int_{t}^{N_{n}(t)}\left(t-S_{n}(s)\right) d s \\
= & \int_{N_{n}(t)}^{t}\left\{\left(S_{n}(s)-s\right)-\left(S_{n}(t)-t\right)\right\} d s-\int_{N_{n}(t)}^{t}(t-s) d s-\left(S_{n}(t)-t\right)\left(N_{n}(t)-t\right)+t^{2} .
\end{aligned}
$$

Consequently, we conclude (2.1) (cf. also Figure 1) as follows:

$$
V_{n}(t)=\int_{0}^{t}\left(\left(S_{n}(s)-s\right)+\left(N_{n}(s)-s\right)\right) d s=A_{n}(t)-\frac{1}{2} \bar{N}_{n}^{2}(t)-\bar{S}_{n}(t) \bar{N}_{n}(t)+t^{2}-t^{2} .
$$




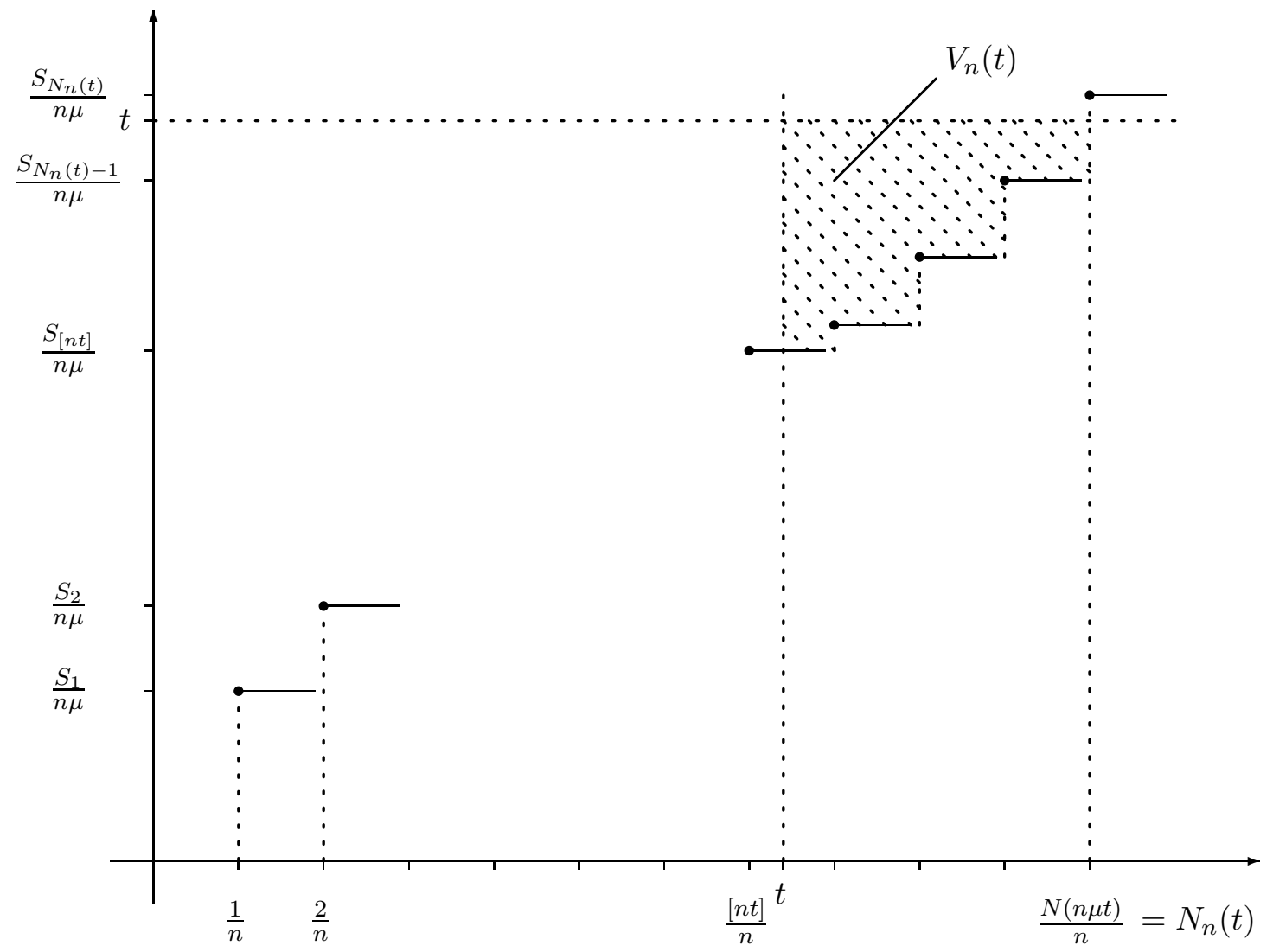

Figure 1

As an immediate consequence in our proof, we get

$$
\begin{aligned}
V_{n}(t)-\frac{1}{2} \bar{S}_{n}^{2}(t) & =A_{n}(t)-\frac{1}{2}\left(\bar{S}_{n}(t)+\bar{N}_{n}(t)\right)^{2} \\
& =A_{n}(t)-\frac{1}{2} M_{n}^{2}(t) .
\end{aligned}
$$

Now, in view of (1.6), (1.12) and the law of the iterated logarithm for a Wiener process,

$$
\left\|M_{n}^{2}\right\|=O\left(n^{-3 / 2}(\log n)(\log \log n)^{1 / 2}\right) \quad \text { a.s. }
$$

and, as a consequence of (1.14),

$$
\left\|M_{n}^{2}\right\|=O_{P}\left(n^{-3 / 2} \log n\right) .
$$

Next, we show that $A_{n}$ is the dominating process on the right-hand side of (2.5). In (2.2) put $s=t-u\left(t-N_{n}(t)\right)=t+u \bar{N}_{n}(t)$. Then we get

$$
A_{n}(t)=-\bar{N}_{n}(t) \int_{0}^{1}\left(\bar{S}_{n}\left(t+u \bar{N}_{n}(t)\right)-\bar{S}_{n}(t)\right) d u .
$$

On account of (1.5),

$$
\frac{n \mu}{\sigma} \bar{S}_{n}(t)=W(n t)+o\left(n^{1 / 4}\right) \quad \text { a.s. }
$$


uniformly in $t \in[0,1]$. A combination of (2.8) and (2.9) yields

$$
\frac{n \mu}{\sigma} A_{n}(t)=-\bar{N}_{n}(t) \int_{0}^{1}\left(W\left(n t+u n \bar{N}_{n}(t)\right)-W(n t)\right) d u+o\left(n^{-1 / 4}(\log \log n)^{1 / 2}\right) \quad \text { a.s. }
$$

uniformly in $t \in[0,1]$, since

$$
n \bar{N}_{n}(t)=O\left((n \log \log n)^{1 / 2}\right) \quad \text { a.s. }
$$

uniformly in $t \in[0,1]$.

This rate in (2.10) can also be replaced by $o_{P}\left(n^{-1 / 4}\right)$, on account of

$$
n \bar{N}_{n}(t)=O_{P}\left(n^{1 / 2}\right)
$$

uniformly in $t \in[0,1]$.

Moreover, by Theorem 1.2.1 of Csörgő and Révész (1981),

$$
W\left(n t+u n \bar{N}_{n}(t)\right)-W(n t)=O\left(n^{1 / 4}(\log n)^{1 / 2}(\log \log n)^{1 / 4}\right) \quad \text { a.s. }
$$

as well as

$$
W\left(n t+u n \bar{N}_{n}(t)\right)-W(n t)=O_{P}\left(n^{1 / 4}(\log n)^{1 / 2}\right),
$$

uniformly in $u, t \in[0,1]$.

In view of (1.6), we also have

$$
\frac{\mu}{\sigma} n \bar{N}_{n}(t)=-W(n t)+O\left(n^{1 / 4}(\log n)^{1 / 2}(\log \log n)^{1 / 4}\right) \quad \text { a.s. }
$$

As a consequence of (2.10)-(2.13), we arrive at

$$
\frac{n \mu}{\sigma} A_{n}(t)=\frac{\sigma W(n t)}{n \mu} \int_{0}^{1}\left(W\left(n t+u n \bar{N}_{n}(t)\right)-W(n t)\right) d u+o\left(n^{-1 / 4}(\log \log n)^{1 / 2}\right) \quad \text { a.s. }
$$

uniformly in $t \in[0,1]$, where this rate can also be replaced by $o_{P}\left(n^{-1 / 4}\right)$.

On making use of (2.13) in combination with Theorem 1.2.1 of Csörgő and Révész (1981), we conclude the following strong and weak invariance principles for $A_{n}(t)$ of (2.2):

$$
\begin{aligned}
(2.15) A_{n}(t) & =\frac{\sigma W(n t)}{n \mu} \int_{0}^{1} \frac{\sigma}{n \mu}\left(W\left(n t-u \frac{\sigma}{\mu} W(n t)\right)-W(n t)\right) d u+o\left(n^{-5 / 4}(\log \log n)^{1 / 2}\right) \text { a.s. } \\
& =\frac{\sigma}{n \mu} \int_{0}^{\frac{\sigma}{n \mu} W(n t)}(W(n t-n x)-W(n t)) d x+o\left(n^{-5 / 4}(\log \log n)^{1 / 2}\right) \quad \text { a.s. } \\
& =\int_{0}^{Y_{n}(t)}\left(Y_{n}(t-x)-Y_{n}(t)\right) d x+o\left(n^{-5 / 4}(\log \log n)^{1 / 2}\right) \quad \text { a.s., }
\end{aligned}
$$

uniformly in $t \in[0,1]$, where $Y_{n}(t):=\frac{\sigma}{n \mu} W(n t)$, and this a.s. rate can also be replaced by $o_{P}\left(n^{-5 / 4}\right)$.

Arguing similarly as in (2.11) and (2.12), we also have

$$
\begin{aligned}
A_{n}(t) & =O\left(n^{-1 / 2}(\log \log n)^{1 / 2} n^{-3 / 4}(\log n)^{1 / 2}(\log \log n)^{1 / 4}\right) \quad \text { a.s. } \\
& =O\left(n^{-5 / 4}(\log n)^{1 / 2}(\log \log n)^{3 / 4}\right) \quad \text { a.s. },
\end{aligned}
$$


and

$$
A_{n}(t)=O_{P}\left(n^{-5 / 4}(\log n)^{1 / 2}\right)
$$

uniformly in $t \in[0,1]$.

Thus, in view of (2.5), (2.6) and (2.16) we conclude (a) of Theorem 2.1, while (2.5), (2.7) and (2.17) result in (b) of Theorem 2.1.

As an immediate consequence of Theorem 2.1 and Theorem A, we get

Corollary 2.1 Assume the conditions (i), (ii), (iii) and (iv). Then, as $n \rightarrow \infty$, on the probability space of Theorem A with $W_{n}$ as in (1.5), we have

$$
\frac{1}{\log \log n}\left\|n V_{n}-\frac{1}{2}\left(\frac{\sigma}{\mu} W_{n}\right)^{2}\right\| \rightarrow 0 \quad \text { a.s. }
$$

and

$$
\left\|n V_{n}-\frac{1}{2}\left(\frac{\sigma}{\mu} W_{n}\right)^{2}\right\| \stackrel{P}{\rightarrow} 0 .
$$

As a consequence of (b) of Corollary 2.1, one concludes also

$$
n V_{n} \stackrel{\mathcal{D}}{\rightarrow} Z, \quad n \rightarrow \infty
$$

in the space $C[0,1]$ (endowed with the uniform topology), where $Z(t):=\frac{1}{2}\left(\frac{\sigma}{\mu} W(t)\right)^{2}, t \in[0,1]$.

The strong invariance principle (a) of Corollary 2.1 in turn implies Strassen (1964)-type laws of the iterated logarithm for $V_{n}$ as follows.

Corollary 2.2 Assume the conditions (i), (ii), (iii) and (iv). Then, as $n \rightarrow \infty$, the set

$$
\left\{\frac{\mu^{2} n V_{n}}{\sigma^{2} \log \log n}, n \geq 3\right\}
$$

is relatively compact in $C[0,1]$, equipped with the sup-norm $\|\cdot\|$. Furthermore, the set of all limit points of the functions in the set (2.19) almost surely coincides with the set $\left\{f^{2}: f \in \mathcal{S}\right\}$, where $\mathcal{S}$ is the Strassen class of all absolutely continuous functions $f$ on $[0,1]$ such that $f(0)=0$ and $\left\|f^{\prime}\right\|_{2} \leq 1$, where $\|\cdot\|_{p}$ denotes the $L_{p}$-norm, $p \geq 1$.

As examples of consequences of Corollary 2.2 à la Strassen (1964), we mention the following results.

Corollary 2.3 Under the conditions (i), (ii), (iii) and (iv) we have

$$
\limsup _{n \rightarrow \infty} \frac{n\left\|V_{n}\right\|}{\log \log n}=\frac{\sigma^{2}}{\mu^{2}} \quad \text { a.s. }
$$

and

$$
\limsup _{n \rightarrow \infty} \frac{n\left\|V_{n}\right\|_{1}}{\log \log n}=\frac{4 \sigma^{2}}{\mu^{2} \pi^{2}} \quad \text { a.s. }
$$




\section{The Vervaat process for general renewals}

In this section we study the case of general renewals for which assumption (iv) of Section 2 may not necessarily be true. In order to do this, we need to introduce some further notations and auxiliary results:

Let $\nu_{i}$ denote the $i$-th (strong) ascending ladder index of the partial sum sequence $\left\{S_{n}\right\}_{n=0,1, \ldots}$, i.e., $\nu_{0}=0$, and, recursively,

$$
\nu_{i}:=\min \left\{n>\nu_{i-1}: S_{n}-S_{\nu_{i-1}}>0\right\} .
$$

We note that, under assumptions (i), (ii), (iii), $\left\{\nu_{i}-\nu_{i-1}\right\}_{i=1,2, \ldots}$ is an i.i.d. sequence of random variables with

$$
E \nu_{1}^{4}<\infty
$$

Moreover, the sequence $\left\{S_{\nu_{i}}-S_{\nu_{i-1}}\right\}_{i=1,2, \ldots}$ of ladder heights is also an i.i.d. sequence of random variables with

$$
E S_{\nu_{1}}^{4}<\infty
$$

(cf., e.g., Gut (1988, Sections III. 2-3)). By definition,

$$
N(t)=\nu_{i}, \quad \text { for } S_{\nu_{i-1}} \leq t<S_{\nu_{i}} \quad(i=1,2, \ldots ; t \geq 0),
$$

i.e.,

$$
N(n \mu s)=\nu_{i}, \quad \text { for } \frac{S_{\nu_{i-1}}}{n \mu} \leq s<\frac{S_{\nu_{i}}}{n \mu} \quad(i=1,2, \ldots ; s \geq 0) .
$$

Let $N(n \mu t)=\nu_{\ell}$, i.e.,

$$
\ell=\min \left\{j: S_{\nu_{j}}>n \mu t\right\},
$$

that is, $\ell=N_{H}(n \mu t)$, where $N_{H}$ denotes the (ordinary) renewal process corresponding to the (ladder height) sequence $S_{\nu_{1}}, S_{\nu_{2}}-S_{\nu_{1}}, \ldots$. This will play a crucial role in the calculations below.

We first note that we continue to use the same definition for the Vervaat process $V_{n}$ for general renewals as in (1.17). Namely, we study the integral

$$
V_{n}(t)=\int_{0}^{t} M_{n}(s) d s=\int_{0}^{t}\left(\bar{S}_{n}(s)+\bar{N}_{n}(s)\right) d s,
$$

where $\bar{S}_{n}$ and $\bar{N}_{n}$ are as in (2.3) and (2.4), respectively, i.e.,

$$
\begin{aligned}
& \bar{S}_{n}(t)=S_{n}(t)-t=(n \mu)^{-1}\left(S_{[n t]}-n \mu t\right), \\
& \bar{N}_{n}(t)=N_{n}(t)-t=n^{-1}(N(n \mu t)-n t),
\end{aligned}
$$

with $S_{n}(t)$ and $N_{n}(t)$ as in (1.1) and (1.3), respectively.

Consider now the following figure. 


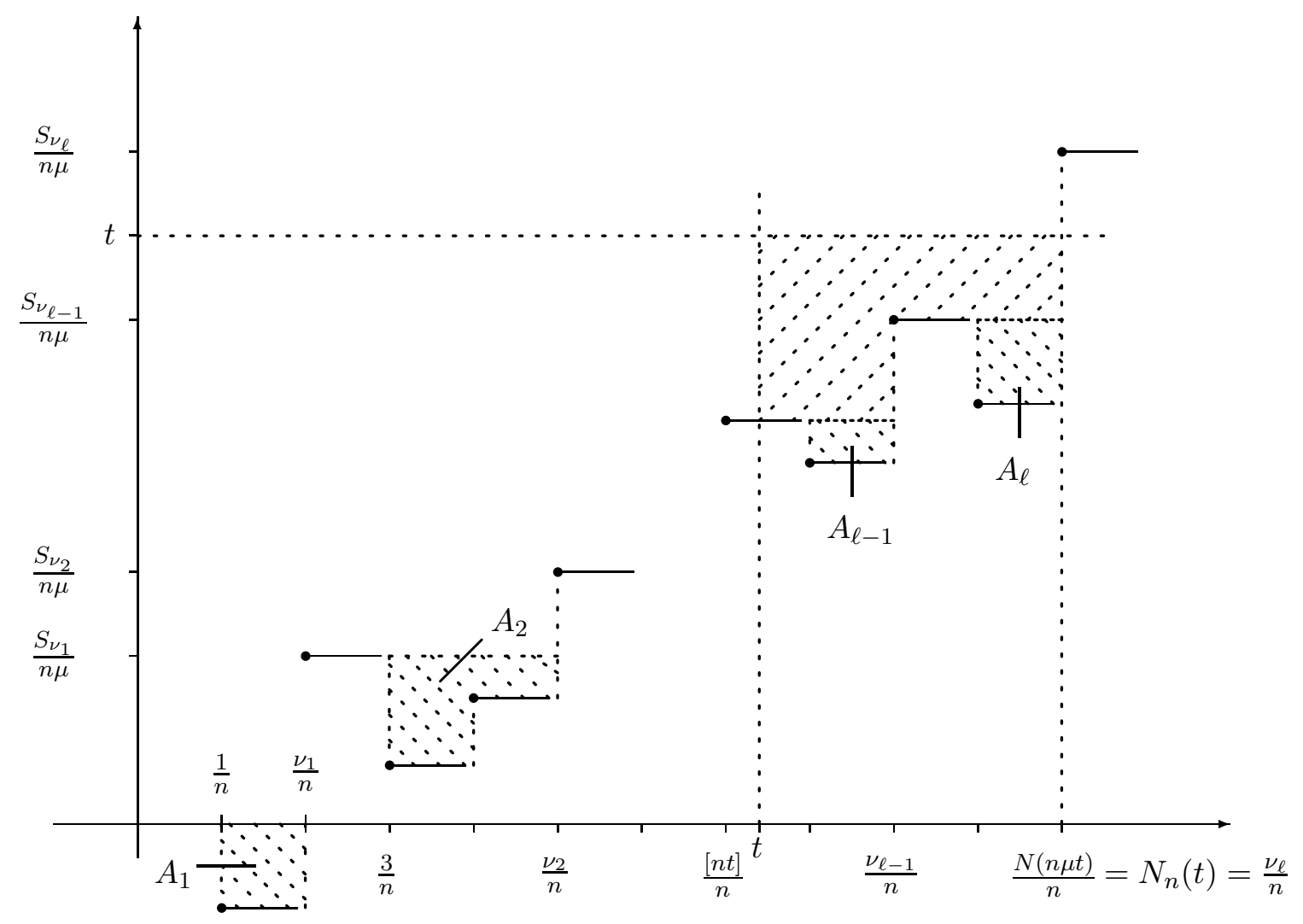

Figure 2

Note that, in contrast to the case of the ordinary renewal process (cf. Figure 1), the (random) areas $A_{1}, A_{2}, \ldots$ (cf. Figure 2) are additional in the present case of the Vervaat process for general renewals. Thus, in computing the above integral $V_{n}$, these areas are to be taken into consideration. Our next lemma accomplishes this via establishing the following new representation for $V_{n}$ in the general case that will now replace that of (2.5).

Lemma 3.1 Under the conditions (i), (ii), (iii) we have

$$
V_{n}(t)=A_{n}(t)+B_{n}(t)-\frac{1}{2} M_{n}^{2}(t)+\frac{1}{2} \bar{S}_{n}^{2}(t),
$$

with $A_{n}$ as in (2.2), $M_{n}=\bar{S}_{n}+\bar{N}_{n}$ and $B_{n}$ is defined by

$$
B_{n}(t):=\frac{1}{n^{2} \mu} \sum_{i=1}^{N_{H}(n \mu t)}\left(\sum_{j=1}^{\nu_{i}-\nu_{i-1}-1}\left(\nu_{i}-\nu_{i-1}-j\right) X_{\nu_{i-1}+j}\right),
$$

with $N_{H}(n \mu t):=\ell$ of $(3.5)$. 
Proof. We first show that

$$
\int_{0}^{t} \bar{N}_{n}(s) d s+\int_{0}^{N_{n}(t)} \bar{S}_{n}(s) d s=-\frac{1}{2} \bar{N}_{n}^{2}(t)+B_{n}(t),
$$

We have

$$
\int_{0}^{t}\left(N_{n}(s)-s\right) d s+\int_{0}^{N_{n}(t)}\left(S_{n}(s)-s\right) d s=\int_{0}^{t} N_{n}(s) d s+\int_{0}^{N_{n}(t)} S_{n}(s) d s-\frac{t^{2}}{2}-\frac{N_{n}^{2}(t)}{2}
$$

By (3.4) and the definition of $N_{n}$ we arrive at

$$
\begin{aligned}
\int_{0}^{t} N_{n}(s) d s & =\frac{1}{n} \sum_{i=1}^{\ell} \int_{\frac{S_{\nu_{i-1}}}{n \mu}}^{\frac{S_{\nu_{i}}}{n \mu}} \nu_{i} d s-\frac{1}{n} \int_{t}^{\frac{S_{\nu_{\ell}}}{n \mu}} \nu_{\ell} d s \\
& =\frac{1}{n^{2} \mu} \sum_{i=1}^{\ell} \nu_{i}\left(S_{\nu_{i}}-S_{\nu_{i-1}}\right)-\frac{1}{n} \nu_{\ell}\left(\frac{S_{\nu_{\ell}}}{n \mu}-t\right)
\end{aligned}
$$

Also,

$$
\begin{aligned}
\int_{0}^{N_{n}(t)} S_{n}(s) d s & =\sum_{i=1}^{\nu_{\ell}} \int_{\frac{i-1}{n}}^{\frac{i}{n}} S_{n}(s) d s=\frac{1}{n^{2} \mu} \sum_{i=1}^{\nu_{\ell}} S_{i-1} \\
& =\frac{1}{n^{2} \mu} \sum_{i=1}^{\ell} \sum_{j=1}^{\nu_{i}-\nu_{i-1}}\left(S_{\nu_{i-1}+j-1}-S_{\nu_{i-1}}\right)+\frac{1}{n^{2} \mu} \sum_{i=1}^{\ell}\left(\nu_{i}-\nu_{i-1}\right) S_{\nu_{i-1}} .
\end{aligned}
$$

Now, for any real sequences $\left\{a_{i}\right\}_{i=0,1, \ldots}$ and $\left\{b_{i}\right\}_{i=0,1, \ldots}$, it can easily be checked that

$$
\sum_{i=1}^{n} a_{i}\left(b_{i}-b_{i-1}\right)+\sum_{i=1}^{n} b_{i-1}\left(a_{i}-a_{i-1}\right)=a_{n} b_{n}-a_{0} b_{0} .
$$

Hence, since $\nu_{0}=0=S_{\nu_{0}}$,

$$
\sum_{i=1}^{\ell} \nu_{i}\left(S_{\nu_{i}}-S_{\nu_{i-1}}\right)=\nu_{\ell} S_{\nu_{\ell}}-\sum_{i=1}^{\ell}\left(\nu_{i}-\nu_{i-1}\right) S_{\nu_{i-1}}
$$

Thus, on combining (3.9) - (3.12), we obtain

$$
\begin{aligned}
\int_{0}^{t} \bar{N}_{n}(s) d s+\int_{0}^{N_{n}(t)} \bar{S}_{n}(s) d s \\
=\frac{1}{n^{2} \mu} \sum_{i=1}^{\ell} \sum_{j=1}^{\nu_{i}-\nu_{i-1}-1}\left(S_{\nu_{i-1}+j}-S_{\nu_{i-1}}\right)-\frac{t^{2}}{2}-\frac{N_{n}^{2}(t)}{2}+t \frac{\nu_{\ell}}{n} \\
=-\frac{1}{2}\left(N_{n}(t)-t\right)^{2}+\frac{1}{n^{2} \mu} \sum_{i=1}^{\ell}\left(\sum_{j=1}^{\nu_{i}-\nu_{i-1}-1}\left(\nu_{i}-\nu_{i-1}-j\right) X_{\nu_{i-1}+j}\right) .
\end{aligned}
$$

This, in turn, proves (3.8).

As a consequence of (3.8) and the definition of $A_{n}(t)$ we now conclude our claim in (3.6) as follows. 


$$
\begin{aligned}
V_{n}(t) & =\int_{0}^{t}\left(\bar{S}_{n}(s)+\bar{N}_{n}(s)\right) d s \\
& =\int_{0}^{t} \bar{N}_{n}(s) d s+\int_{0}^{N_{n}(t)} \bar{S}_{n}(s) d s+\int_{N_{n}(t)}^{t} \bar{S}_{n}(s) d s \\
& =-\frac{1}{2} \bar{N}_{n}^{2}(t)+B_{n}(t)+A_{n}(t)-\bar{S}_{n}(t) \bar{N}_{n}(t) \\
& =A_{n}(t)+B_{n}(t)-\frac{1}{2} M_{n}^{2}(t)+\frac{1}{2} \bar{S}_{n}^{2}(t) .
\end{aligned}
$$

For the sake of stating and proving an analogue of Theorem 2.1 in case of the general renewal process, we need further notations.

Consider the summands

$$
D_{i}:=\sum_{j=2}^{\nu_{i}-\nu_{i-1}}\left(\nu_{i}-\nu_{i-1}-j\right) X_{\nu_{i-1}+j-1} \quad i=1,2, \ldots
$$

in the definition of $B_{n}$ (cf. 3.7). We note that the just introduced random variables $D_{i}$ are i.i.d. with mean $\mu_{D}:=E D_{1}$ and finite second moment $E D_{1}^{2}<\infty$. The latter is implied by (3.2) and (3.3), on applying the Cauchy-Schwarz inequality when computing $E D_{1}^{2}$. Put also $\mu_{H}:=E S_{\nu_{1}}$, the so-called expected ladder height (cf., e.g., Gut (1988, Chap. IV, Theorem 2.7)).

The extension of our Theorem 2.1 to the general renewal situation now reads as follows.

Theorem 3.1 Assume the conditions (i), (ii), (iii). Then, as $n \rightarrow \infty$,

$$
\begin{gathered}
\frac{1}{\log \log n}\left\|n V_{n}-\frac{n}{2} \bar{S}_{n}^{2}-\left(\frac{\mu_{D}}{\mu_{H}}\right) i d\right\| \rightarrow 0 \quad \text { a.s. } \\
\left\|n V_{n}-\frac{n}{2} \bar{S}_{n}^{2}-\left(\frac{\mu_{D}}{\mu_{H}}\right) i d\right\| \stackrel{P}{\rightarrow} 0
\end{gathered}
$$

where $i d(t):=t, t \in[0,1]$.

Proof. For $B_{n}$ of Lemma 3.1 we conclude the following asymptotic representation. As $n \rightarrow \infty$, by the law of the iterated logarithm, we have almost surely

$$
\begin{aligned}
B_{n}(t) & =\frac{1}{n^{2} \mu} \sum_{i=1}^{N_{H}(n \mu t)} D_{i} \\
& =\frac{1}{n^{2} \mu}\left(\sum_{i=1}^{N_{H}(n \mu t)} D_{i}-n \mu t \frac{\mu_{D}}{\mu_{H}}\right)+\frac{1}{n}\left(\frac{\mu_{D}}{\mu_{H}}\right) t \\
& =\frac{1}{n}\left(\frac{\mu_{D}}{\mu_{H}}\right) t+O\left(\frac{(\log \log n)^{1 / 2}}{n^{3 / 2}}\right)
\end{aligned}
$$


uniformly in $t \in[0,1]$. Consequently, via (2.6) / (2.7), (2.16) / (2.17), Lemma 3.1 and (3.15) we conclude the respective two statements of Theorem 3.1 .

Remark 3.1 Via the definition of the ladder index $\nu_{1}$, we have

$$
X_{1}, X_{1}+X_{2}, \ldots, X_{1}+\cdots+X_{\nu_{1}-1} \leq 0, X_{1}+\cdots+X_{\nu_{1}}>0
$$

Hence (cf. (3.14)) we have also

$$
D_{1}=\sum_{j=1}^{\nu_{1}-1}\left(\nu_{1}-j\right) X_{j} \leq 0
$$

If, however,

$$
P\left(X_{1} \geq 0\right)=1
$$

then

$$
P\left(D_{1}=0\right)=1 \text {. }
$$

On the other hand, if

$$
P\left(X_{1}<0\right)>0
$$

then we have

$$
P\left(D_{1}<0\right)>0
$$

as well.

This shows that the results of Section 2 also remain true under assumption (iv') replacing (iv), for then $B_{n}=0$ a.s.

As an immediate consequence of Theorem 3.1 and Theorem A, we conclude

Corollary 3.1. Assume the conditions (i), (ii), (iii). Then, as $n \rightarrow \infty$, on the probability space of Theorem A with $W_{n}$ as in (1.5), we have

$$
\frac{1}{\log \log n}\left\|n V_{n}-\frac{1}{2}\left(\frac{\sigma}{\mu} W_{n}\right)^{2}-\left(\frac{\mu_{D}}{\mu_{H}}\right) i d\right\| \rightarrow 0 \quad \text { a.s. }
$$

and

$$
\left\|n V_{n}-\frac{1}{2}\left(\frac{\sigma}{\mu} W_{n}\right)^{2}-\left(\frac{\mu_{D}}{\mu_{H}}\right) i d\right\| \stackrel{P}{\rightarrow} 0
$$

As a consequence of (b) of Corollary 3.1, we conclude also

$$
n V_{n} \stackrel{\mathcal{D}}{\rightarrow} \widetilde{Z}, \quad n \rightarrow \infty
$$

in the space $C[0,1]$ (endowed with the uniform topology), where $\widetilde{Z}(t):=\frac{1}{2}\left(\frac{\sigma}{\mu} W(t)\right)^{2}+\left(\frac{\mu_{D}}{\mu_{H}}\right) t$, $t \in[0,1]$. 
The latter conclusion in turn also allows us a Vervaat (1972)-type proof of Corollary 1.1. Namely, if the statement (1.15) were true, then the sequence in there could be replaced by $\{n\}$, due to the convergence of types theorem (cf. Lemma 1 on p. 253 of Feller (1971)). But then one would also have the equality in distribution

$$
\int_{0}^{t} Y(s) d s \stackrel{\mathcal{D}}{=} \frac{1}{2}\left(\frac{\sigma}{\mu} W(t)\right)^{2}+\left(\frac{\mu_{D}}{\mu_{H}}\right) t, \quad t \in[0,1]
$$

which is impossible, since the Wiener process $W$ is a.s. nowhere differentiable. Consequently, the relation (1.15) cannot hold true.

In concluding this section, it may be of interest to note that, while it is true that the asymptotic behaviour of the Bahadur-Kiefer-type process $R_{n}^{*}$ of (1.12) with ordinary and general renewals is the same pointwise and in sup-norm, their integrated form $V_{n}$ behaves differently as $n \rightarrow \infty$. Namely, in Corollary 3.1 and (3.18) the squared Wiener process appears with a drift term which is not present in Corollary 2.1 and (2.18).

\section{Vervaat-error type processes for partial sums and renewals}

Theorem 2.1 and Theorem 3.1 suggest the study of the asymptotic behaviour of the following Vervaaterror type process:

$$
Q_{n}(t):=V_{n}(t)-\frac{1}{2} \bar{S}_{n}^{2}(t)-\left(\frac{\mu_{D}}{\mu_{H}}\right) t, \quad 0 \leq t \leq 1 .
$$

We note that the drift term $\left(\mu_{D} / \mu_{H}\right) t$ is identically zero in case of an ordinary renewal process (cf. Theorem 2.1).

Define the stochastic process $Z_{n}$ by

$$
Z_{n}(t):=\int_{0}^{Y_{n}(t)}\left(Y_{n}(t-x)-Y_{n}(t)\right) d x, \quad 0 \leq t \leq 1,
$$

where $Y_{n}(t):=\frac{\sigma}{n \mu} W(n t)$.

We have the following representations for $Q_{n}$ in terms of $Z_{n}$.

Proposition 4.1 Assume the conditions (i), (ii), (iii). Then, as $n \rightarrow \infty$, on the probability space of Theorem A, we have

$$
Q_{n}(t)=Z_{n}(t)+o\left(n^{-5 / 4}(\log \log n)^{1 / 2}\right) \quad \text { a.s. }
$$

and

$$
Q_{n}(t)=Z_{n}(t)+o_{P}\left(n^{-5 / 4}\right)
$$

uniformly in $t \in[0,1]$.

Proof. In view of (3.6), via (2.15), (3.15), and (2.6), we conclude (4.3). Similarly, and again in view of (3.6), via (2.15) with the error term $o_{P}\left(n^{-5 / 4}\right)$, (3.15), and (2.7), we arrive at (4.4) as well. 
Theorem 4.1 Assume (i), (ii), (iii). Then, for $0<t \leq 1$ fixed, as $n \rightarrow \infty$

$$
n^{5 / 4} Q_{n}(t) \stackrel{\mathcal{D}}{\rightarrow}\left(\frac{1}{3}\right)^{1 / 2}\left(\frac{\sigma}{\mu}\right)^{5 / 2} t^{3 / 4} \mathcal{N}|\tilde{\mathcal{N}}|^{3 / 2},
$$

where $\mathcal{N}$ and $\tilde{\mathcal{N}}$ are independent standard normal random variables.

We note in passing that the result of (4.5) parallels that of (2.5) of Theorem 2.1 of Csáki et al. (2002), which deals with a similar problem concerning the Vervaat-error process of the empirical and quantile processes. For further related results along these lines we refer to Csörgő and Zitikis (1999).

Proof. Given (4.4) of Proposition 4.1, it is clear that the pointwise asymptotic distribution of $Q_{n}(t)$ for any fixed $t \in(0,1]$ can be established via that of $Z_{n}(t)$. Clearly, from (4.2) we obtain

$$
Z_{n}(t)=\frac{\sigma W(n t)}{n \mu} \int_{0}^{1} \frac{\sigma}{n \mu}\left(W\left(n t-s \frac{\sigma}{\mu} W(n t)\right)-W(n t)\right) d s .
$$

Let

$$
\begin{aligned}
& W_{1}(u):=(n t)^{-1 / 2}(W(n t-u n t)-(1-u) W(n t))+u \mathcal{N}^{*}, \quad 0 \leq u \leq 1, \\
& W_{2}(u):=(n t)^{-1 / 2}(W(n t+u n t)-W(n t)), \quad 0 \leq u \leq 1
\end{aligned}
$$

where $\mathcal{N}^{*}$ is a standard normal random variable, independent of $W(\cdot)$. It is obvious that for any fixed $n$ and $t$, the processes $\left\{W_{1}(u), 0 \leq u \leq 1\right\}$ and $\left\{W_{2}(u), 0 \leq u \leq 1\right\}$ are independent standard Wiener processes, which are also independent of $W(n t)$.

Consider $Z_{n}(t)$ of (4.6), and first assume that $W(n t)>0$. Put unt $=s(\sigma / \mu) W(n t)$ in (4.6). Thus we obtain

$$
\begin{gathered}
Z_{n}(t)=\frac{t \sigma}{n \mu} \int_{0}^{\frac{\sigma W(n t)}{\mu n t}}(W(n t-u n t)-W(n t)) d u \\
=\frac{t \sigma}{\mu}\left(\frac{t}{n}\right)^{1 / 2} \int_{0}^{\frac{\sigma W(n t)}{\mu n t}} W_{1}(u) d u-\frac{t \sigma}{\mu n} \int_{0}^{\frac{\sigma W(n t)}{\mu n t}} u W(n t) d u-\frac{t \sigma}{\mu}\left(\frac{t}{n}\right)^{1 / 2} \int_{0}^{\frac{\sigma W(n t)}{\mu n t}} u \mathcal{N}^{*} d u \\
=\frac{t \sigma}{\mu}\left(\frac{t}{n}\right)^{1 / 2} \int_{0}^{\frac{\sigma W(n t)}{\mu n t}} W_{1}(u) d u+O_{P}\left(n^{-3 / 2}\right) .
\end{gathered}
$$

Assuming now that $W(n t)<0$, on putting unt $=-s(\sigma / \mu) W(n t)$ in (4.6), we get

$$
Z_{n}(t)=\frac{t \sigma}{\mu}\left(\frac{t}{n}\right)^{1 / 2} \int_{0}^{\frac{-\sigma W(n t)}{\mu n t}} W_{2}(u) d u .
$$

Hence, for each fixed $t \in(0,1], Z_{n}(t)$ has the following representation

$$
Z_{n}(t)=Z_{n}^{*}(t)+O_{P}\left(n^{-3 / 2}\right)
$$

where

$$
Z_{n}^{*}(t):=\frac{t \sigma}{\mu}\left(\frac{t}{n}\right)^{1 / 2} \int_{0}^{\left|\frac{\sigma W(n t)}{\mu n t}\right|} W^{*}(u) d u,
$$

and $W^{*}(u):=W_{1}(u) I\{W(n t)>0\}+W_{2}(u) I\{W(n t)<0\}$ is a standard Wiener process, independent of $W(n t)$ for each fixed $n$ and $t$. 
It is well-known that for any fixed $T>0$,

$$
\int_{0}^{T} W^{*}(u) d u \stackrel{\mathcal{D}}{=} N\left(0, \frac{T^{3}}{3}\right) \stackrel{\mathcal{D}}{=} \frac{T^{3 / 2}}{3^{1 / 2}} N(0,1) .
$$

Consequently, by independence of $W(n t)$ and $\left\{W^{*}(u), 0 \leq u \leq 1\right\}$ for each fixed $n$ and $t$,

$$
Z_{n}^{*}(t) \stackrel{\mathcal{D}}{=}\left(\frac{1}{3}\right)^{1 / 2}\left(\frac{\sigma}{\mu}\right)^{5 / 2} \frac{1}{n^{2}}\left(\frac{|W(n t)|}{(n t)^{1 / 2}}\right)^{3 / 2}(n t)^{3 / 4} \mathcal{N} .
$$

Hence for each fixed $n$ and $t$ we conclude

$$
Z_{n}^{*}(t) \stackrel{\mathcal{D}}{=} n^{-5 / 4}\left(\frac{1}{3}\right)^{1 / 2}\left(\frac{\sigma}{\mu}\right)^{5 / 2} t^{3 / 4}|\tilde{\mathcal{N}}|^{3 / 2} \mathcal{N}
$$

where $\mathcal{N}$ and $\widetilde{\mathcal{N}}$ are independent standard normal random variables.

Consequently, on account of (4.7), as $n \rightarrow \infty$, we obtain

$$
n^{5 / 4} Z_{n}(t) \stackrel{\mathcal{D}}{\rightarrow}\left(\frac{1}{3}\right)^{1 / 2}\left(\frac{\sigma}{\mu}\right)^{5 / 2} t^{3 / 4} \mathcal{N}|\tilde{\mathcal{N}}|^{3 / 2},
$$

for each fixed $t \in(0,1]$.

This in view of (4.4) of Proposition 4.1 also completes the proof of (4.5) of Theorem 4.1.

\section{References}

[1] Bahadur, R.R. (1966). A note on quantiles in large samples. Ann. Math. Statist. 37 577-580.

[2] Csáki, E., Csörgö, M., Földes, A., Shi, Z., and Zitikis, R. (2002). Pointwise and uniform asymptotics of the Vervaat error process. J. Theor. Probab. 15 845-875.

[3] Csörgö, M., and Révész, P. (1981). Strong Approximations in Probability and Statistics. Academic Press, New York.

[4] Csörgö, M., and Horváth, L. (1993). Weighted Approximations in Probability and Statistics. Wiley, Chichester.

[5] Csörgő, M., and Zitikis, R. (1999). On the Vervaat and Vervaat-error processes. Acta Applicandae Mathematicae 58 91-105.

[6] Deheuvels, P., and Mason, D.M. (1990). Bahadur-Kiefer-type processes. Ann. Probab. 18 669-697.

[7] Feller, W. (1971). An Introduction to Probability Theory and Its Applications. Vol. 2 (Second edition), Wiley, New York.

[8] Gut, A. (1988). Stopped Random Walks. Springer, New York.

[9] Horváth, L. (1984). Strong approximations of renewal processes. Stoch. Process. Appl. $18127-$ 138. 
[10] Kiefer, J. (1967). On Bahadur's representation of sample quantiles. Ann. Math. Statist. 38 1323-1342.

[11] Kiefer, J. (1970). Deviations between the sample quantile process and the sample df. In: Nonparametric Techniques in Statistical Inference (ed. M.L. Puri) pp. 299-319. Cambridge University Press, Cambridge.

[12] Komlós, J., Major, P., and Tusnády, G. (1976). An approximation of partial sums of independent R.V.'s and the sample DF. II. Z. Wahrsch. Verw. Gebiete 34 33-58.

[13] Shorack, G.R. (1982). Kiefer's theorem via the Hungarian constructions. Z. Wahrsch. Verw. Gebiete 61 369-373.

[14] Strassen, V. (1964). An invariance principle for the law of the iterated logarithm. Z. Wahrsch. Verw. Gebiete 3 211-226.

[15] Vervaat, W. (1972). Functional central limit theorems for processes with positive drift and their inverses. Z. Wahrsch. Verw. Gebiete 23 245-253. 